\title{
Study of wavelets using chaotic map on DWT based blind video watermarking
}

\author{
Bhavna Sharma $^{1^{*}, \text { Pankaj Sharma }^{2} \text { and Vyom Kulshreshtha }}{ }^{3}$ \\ M.Tech Scholar, Department of Computer Science \& Engineering, Sachdeva Institute of Technology, Mathura, \\ Uttar Pradesh, India ${ }^{1}$ \\ Professor, Department of Computer Science \& Engineering, Sachdeva Institute of Technology, Mathura, Uttar \\ Pradesh, India ${ }^{2}$ \\ Assistant Professor, Department of Computer Science \& Engineering, Sachdeva Institute of Technology, Mathura, \\ Uttar Pradesh, India ${ }^{3}$
}

\section{(C)2017 ACCENTS}

\begin{abstract}
In the past decade there has been rapid growth in distribution and transmission of digital data. Due to easy access to internet and all the digital media such as audios, images, digital documents and videos, there arises the concern over copyright protection of digital content. As an effective solution to this problem, concept of digital watermarking has been used. Watermarks can be of the form images, text, binary logos, signatures, and numbers. They are used for storing information about the copyright owner, source of data, and authentic users. In the proposed work, video watermarking technique has been proposed which highlights comparative analysis of Daubechies (db) wavelets based on different quality parameters. Each of the db wavelets is applied on the randomly selected frames from the input coloured video using random number generated by chaotic logistic map that works as a key for the proposed extraction algorithm. The study of proposed work shows that not all db wavelets support watermarking scheme. Out of 45 wavelets, 10 db wavelets were applicable for watermarking. The original watermark image and the extracted watermark image are then used against various quality parameters to check if the imperceptibility of the watermark is retained after watermark extraction. The proposed watermarking scheme is robust against various quality parameters such as peak-signal-to-noise ratio, mean-square error, maximum difference, and normalized absolute error.
\end{abstract}

\section{Keywords}

Digital watermarking, DWT, Chaotic sequence, Logistic map.

\section{Introduction}

With the advent of internet and high speed networks it become easy and simple to share multimedia data over internet .Internet downloading is also easily achievable. It poses threat to copyright data. As it enhances the illegal copying, modification of content and distribution of data. In this situation there arises a need to secure the original digital content from unauthorized access, duplication and tampering of data. Digital watermarking is the technique which serves the purpose of copyright protection. Digital watermarking provides security, authorization and copyright protection to the digital content. In digital watermarking, additional information such as text, image is embedded into the host image by making some changes in the pixel values. Watermarking technique contains the information about author, legal users of the content.

*Author for correspondence 181
This additional information is embedded permanently in digital content known as watermarks. The benefit of this technique is that the watermark can be extracted at any time from the watermarked object to claim the ownership of the related multimedia contents [1]. In order to be a effective watermark, it should meet three requirements: (i) robustness-it should resist various possible attacks (ii) transparency- it is the perceptual similarity between original and watermark cover image (iii) capacity-it should carry the enough information that represents the uniqueness of data, the more the capacity the more private information can be carried by the host image. Digital watermarking is carried out in three steps: i) embedding ii) Noise /Attack iii) detection. In the first step the watermark is embedded in the host data with some secret key. In digital watermarking the position where watermark is placed is known as host or cover data. The cover data can be anything such as image, text audio. Watermarking system is shown in Figure 1. 


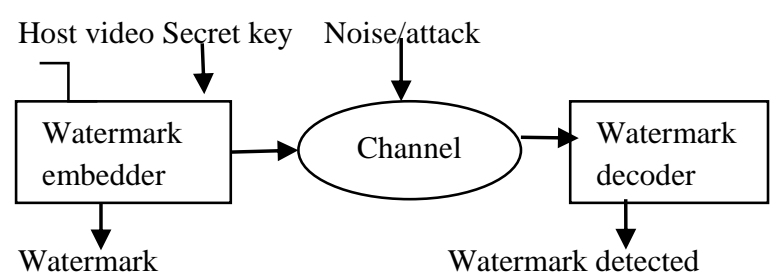

Figure 1 A typical watermarking system

\section{Video watermarking}

A video sequence constructs multiple consecutive frames. Each frame is considered as image. So image watermarking can be extended as video watermarking The main concern with the video watermarking is to decide either the watermark should apply on compressed form or uncompressed form .Videos in electronic devices are generally present in compressed form (mp3, mpeg) .

\subsection{Watermarking techniques}

Digital watermarking is emerged as most promising and efficient solution for security of digital content and copyright protection. Several approaches and method are proposed. Some methods use spatial methods while some use transform domain. The other methods are content based and template based [2]. Simple watermark use spatial watermark by modifying some pixel values. For more robust watermark transform domain is used where watermark is embedded into part of the transformdomain coefficients. Transform domain increases the imperceptibility, robustness and security. There are three main transform methods DWT, DCT, DFT domain.

\subsection{Discrete wavelet transform}

It is multiresolution decomposition of image. The multiresolution representation provides a framework to understand image information where the decoding can be processed sequentially from a low resolution to the higher resolution [3, 4]. DWT is basically based on wavelets which are small waves with small varying frequencies. This is a frequency domain technique in which the cover image is transformed into the frequency domain and then its frequency coefficients are modified in accordance with the transformed coefficients of the watermark and thus watermarked image obtained is more robust [5]. The wavelet transform decomposes the images in three spatial directions i.e. horizontal, vertical and diagonal. It decomposes the image into sub image of spatial domain and independent frequency sub bands. The discrete wavelet transform divides the image in the four frequency components (LL, HL, LH, HH) of low and high levels where first letter corresponds to the low or high pass operation of rows while the second letter corresponds to the operation applied on columns [6]. The low level contains the coarse information and high level contains information about edge components [7]. The LL is the low levels while all the three are higher levels. High frequency range contains the information about the edge components while low frequency levels are further subdivided into low and high frequency parts. LL band happened to have more signal energy thus it is used for embedding the watermark. Figure 2 shows the 1-level DWT.

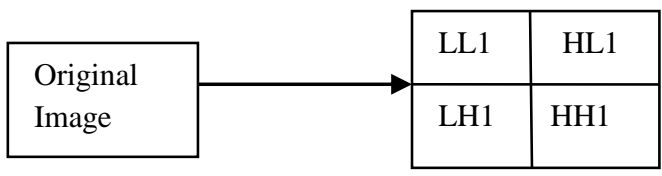

Figure 2 1-Level DWT

After applying 1- level decomposition DWT there is four sub bands LL1, LH1, HL1, and HH1. For each successive level the LL band of previous level is used. For performing 2-DWT, the LL1 band is used and to perform 3-level DWT the LL2 band of 2-level DWT is used 3-level DWT is shown below in Figure 3.

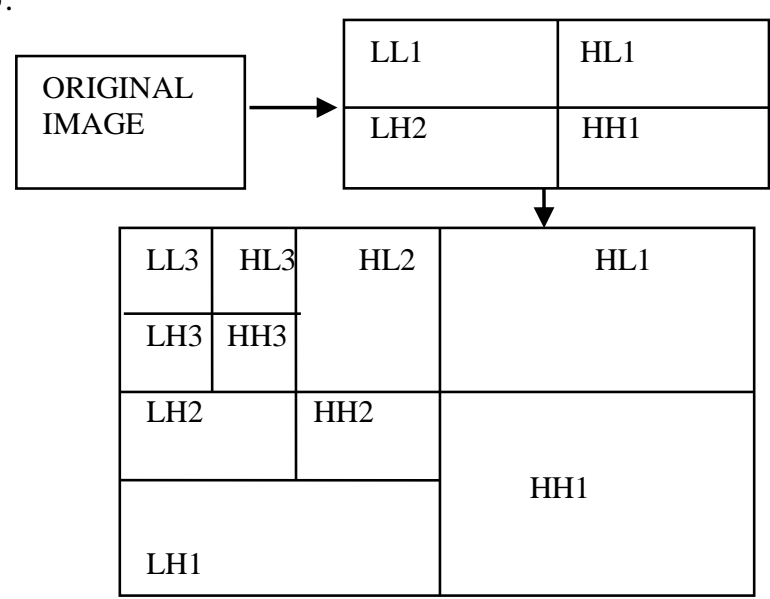

Figure 3 3-Level DWT

\subsection{Chaotic sequence and logistic map}

Chaos Inherent unpredictability or random in the behaviour expressed is by defined system is quasirandom movement that seemingly is irregular of a complex natural system viz. the atmosphere, the beating heart. Chaos system can generate large number of random like high security keys because of very large period and great random of chaos signal, but it is certain. Data embedding is the most attractive features of chaos is extreme sensitivity to 
initial conditions which means that two nearby trajectories starting from initial states diverge exponentially when the time goes to infinity. Complex and unpredictable signals can be easily generated by logistic map. A large number of uncorrelated, random-like, yet deterministic chaotic signals can be generated with small perturbation of parameters. Keeping the chaotic parameters and initial condition as the secret key, the chaotic signal can be reproduced easily [8].

\section{Logistic Map}

Chaotic maps are used in watermarking to increase the security [9]. Logistic map is the simplest chaotic map described by

$X_{\mathrm{k}+1}=\mu \mathrm{X}_{\mathrm{k}}\left(1-\mathrm{X}_{\mathrm{k}}\right)$

Where $0 \leq \mu \leq 4$. When $n 3.5699456<\leq 4$, the map is in the chaotic state. All the sequences generated by the logistic map are very sensitive to initial conditions, in the sense that two logistic sequences generated from different initial conditions are uncorrelated statistically. Moreover, all the orbits of the logistic map are dense in the range of the map [0, 1]. A sequence generated by the logistic map was used once and directly, as the watermark, in [10]. Figure 4 shows the bifurcation diagram of a logistic map.

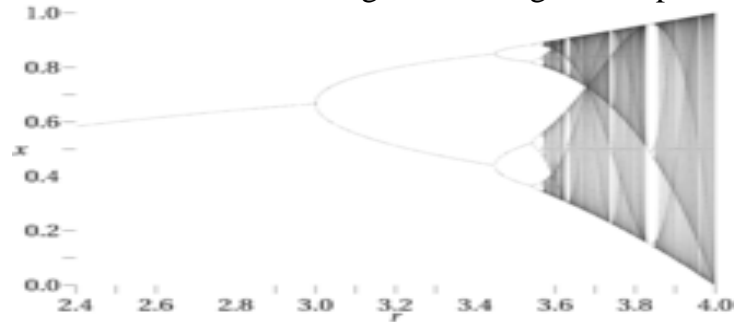

Figure 4 Bifurcation diagram of a logistic map

The chaotic state is displayed by shaded area. In this state, the resulting values appear random, even though the system deterministic Because of its random behavior, a chaotic system can be used as a pseudo-random generator. Hence, initial value of Logistic Map, $x 0$, and constant $\mu$ serve secret keys. When we iterate equation (1) from an initial value (x0) and generate random sequence [11].

\section{The proposed scheme}

In this paper a robust digital video watermarking technique is proposed. The proposed technique watermarking technique applies a colored watermark image is embedded into a video strip using $\mathrm{db}$ wavelets from discrete wavelet transformation family in frequency domain. The binary watermark is embedded in the video frames. The Logistic map is used to detect the suitable position for embedding watermark in the frame. The proposed watermarking algorithm has three steps; one is to embed the watermark into the original video frame, second is to generate the random number and the third to extract the watermark from the received watermarked version of the frame.

\subsection{Watermark embedding algorithm}

The embedding procedure involves various steps as mentioned below:

\section{Input video}

Step1: Read the host video and divide the video into frames.

Step2: Split each of the frames into Red, Green and Blue components.

Step3: Convert the RGB components of each of the frames into Grey components.

Step4: Apply random number generator to select random set of frames for watermark embedding.

\section{Step5: Design a random number generator:}

5.1 Enter a floating point value, $\mathrm{X}$ to the system.

5.2 Convert this value into 32-bit binary number.

5.3 Split the number into 4 equal parts having 6-bit each: X1, X2, X3 and X4.

5.4 Perform Ex-OR operation between ASCII numbers: $\mathrm{Xin}=(\mathrm{X} 1 \mathrm{X} 2) \oplus(\mathrm{X} 3 \mathrm{X} 4)$.

5.5 Perform the operation $\mathrm{X} 0=\mathrm{Xin} / 216$

Using chaotic logistic map

5.6 Use the Chaotic Logistic map to generate a random sequence by:

$\mathrm{X} n+1=\mu \mathrm{Xn}(1-\mathrm{X} n)$

Where $0 \leq \mu \leq 4$, where it shows most chaotic nature at $\mu=4$.Consider the constant value $\mu=3.99$.

Use $\mathrm{X} 0$ as computed in step 4.5 to compute $\mathrm{X} 1$.

5.7 Use this number generated by random number generator to select random set of frames for watermark embedding.

Step6: Apply discrete wavelet transformation using $\mathrm{db}$ wavelets to each of the randomly selected frames in order to obtain four frequency sub bands, LL1, HL1, LH1 and HH1.

Step7: Now take the LL1 frequency sub band and apply second level DWT to obtain LL2, HL2, LH2 and $\mathrm{HH} 2$.

Step8: Again take LL2 frequency sub band and apply third level DWT to obtain LL3, HL3, LH3 and HH3.

\section{Watermark embedding}

Step9: Take the watermark image which is to be embedded in the host video frames and split it into red, blue and green components.

Step10: Convert the RGB components of the watermark image into grey component. 
Step11: Apply wavelet transformation using $\mathrm{db}$ wavelets up to three level decomposition to obtain sub-bands LL3, HL3, LH3 and HH3.

Step12: The watermark bits are then embedded into significant coefficients of the host video frames.

\section{Reconstruction of video}

Step13: Apply Inverse DWT to obtain the watermarked frames.

Step14: Reconstruct the watermarked video by combining frames.

\subsection{Watermark extraction algorithm}

The extraction procedure watermark is extracted from the host video in which it was embedded. Following steps are involved in extraction process

\section{Input Video}

Step1: Read the watermarked video and split it into frames.

Step2: Convert the red, blue and green components into grey component.

\section{Apply 3-level DWT}

Step3: Apply wavelet transformation using $\mathrm{db}$ wavelets up to three level decomposition to obtain sub-bands LL3, HL3, LH3 and HH3.

\section{Extraction}

Step4: Evaluate the difference between the LL3 frequency sub band of watermarked frame and the third level decomposed LL3 frequency sub band of the host frame to get the watermark. Figure 5 shows the extraction algorithm.

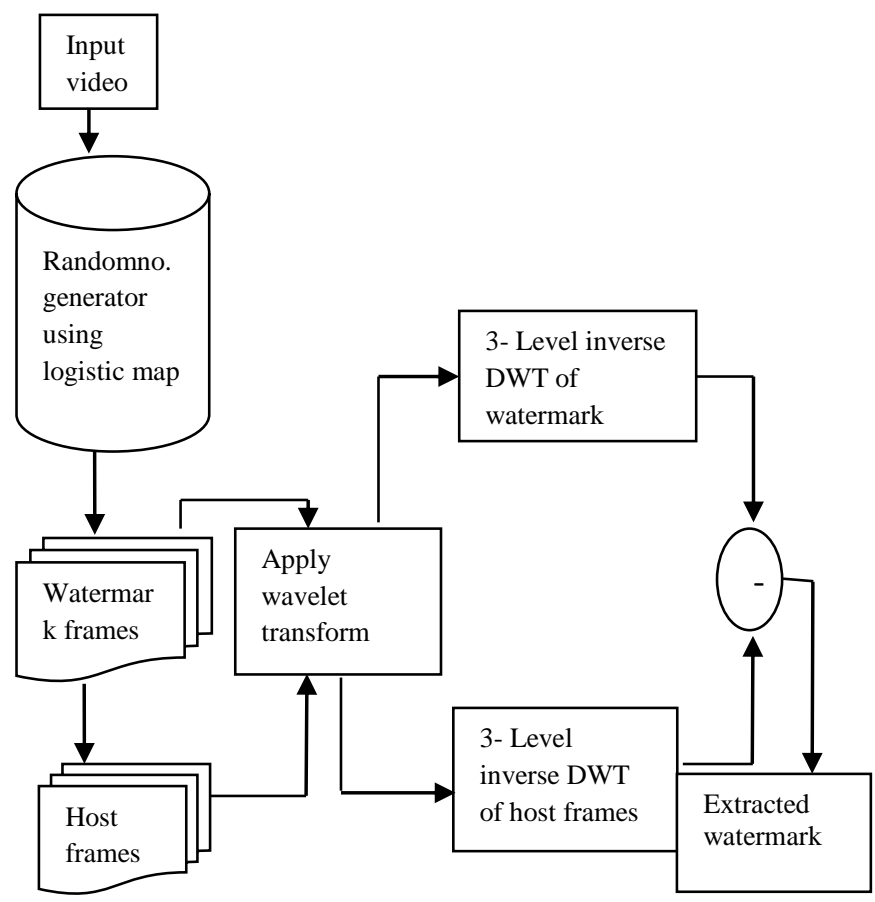

Figure 5 Watermark extraction algorithm

\section{Experimental results}

In the project, the experiments are carried out by using Matlab coding. The inputs are coloured video of avi type and a coloured watermark image that will be embedded inside the video frames. All the frames are of same size. The watermark image used is of the same size of rows and columns as the video frames. The proposed technique uses a chaotic logistic map that generates a set of numbers that will be used as to select the frame number that will be further used for watermark embedding and a secret key at the time of watermark extraction. The proposed algorithm is executed for each of the $\mathrm{db}$ wavelet from $\mathrm{db} 1$ to $\mathrm{db} 45$.The proposed algorithm is executed for each of the $\mathrm{db}$ wavelet from $\mathrm{db} 1$ to $\mathrm{db} 45$. It is observed that $10 \mathrm{db}$ wavelets support the watermarking scheme, namely db45, db41, db37, db29, db25, db21, db13, $\mathrm{db} 9, \mathrm{db} 5, \mathrm{db} 1$. The watermark image and the extracted watermark image are used as input to various quality parameters.

The original host video and watermark image used is shown below in Figure 6.
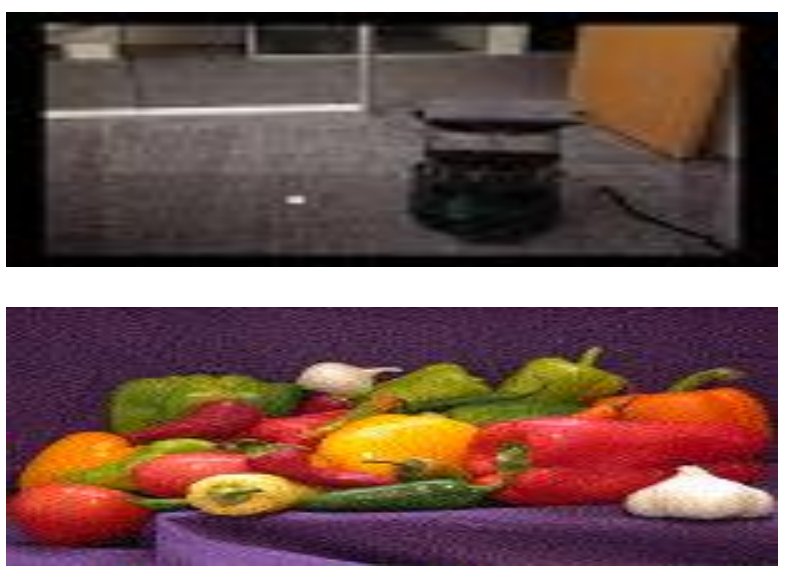

Figure 6 Original host video and watermark image

After 3 level DWT applied on randomly selected frames, the output is shown below in Figure 7.
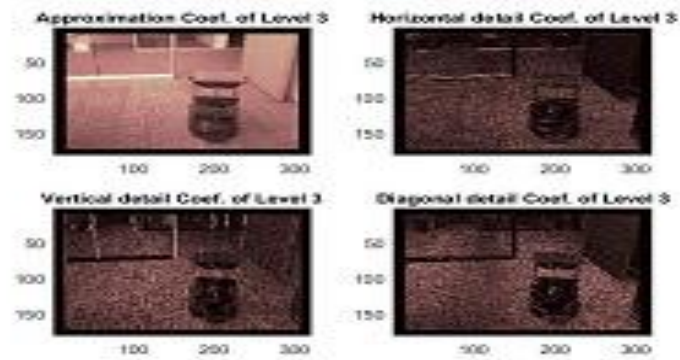

Figure 7 Results after 3-level DWT 
The frame obtained after embedding watermark is shown below in Figure 8.

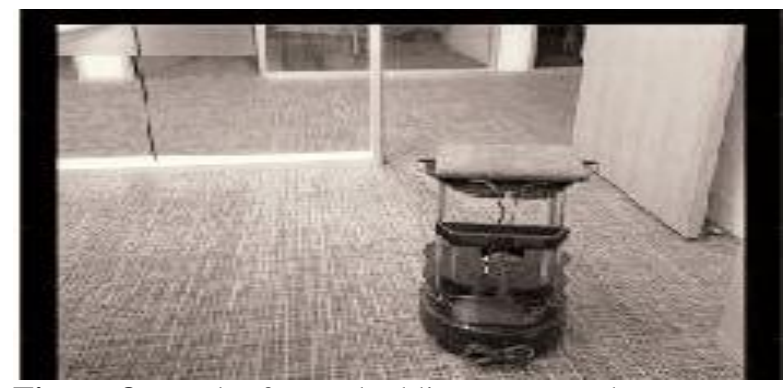

Figure 8 Result after embedding watermark

After applying the watermark extraction algorithm, the extracted watermark is shown below in Figure 9.

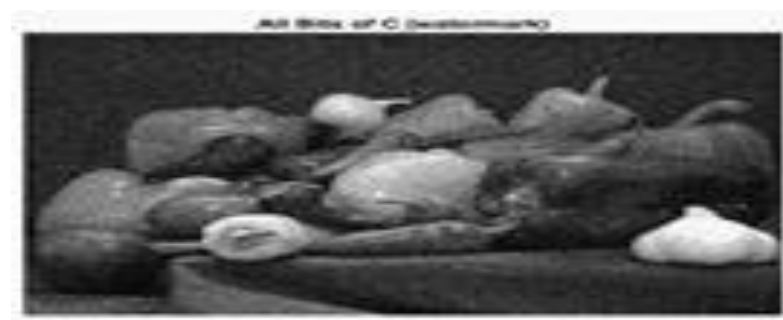

Figure 9 Result after watermark extraction algorithm

\subsection{Quality parameters}

Following quality parameters are used to compare the quality of original watermark and extracted watermark.

4.1.1Mean square error

It is defined as,

$$
\begin{gathered}
M S E=\frac{1}{m n} \sum_{i=0}^{m-1} \sum_{i=0}^{n-1}[I(i, j)-K(i, j)] \\
\text { Where, } \mathrm{I}=\text { the original data } \\
\mathrm{K}=\text { the data after error addition }
\end{gathered}
$$

Larger value of MSE implies the poor quality of video

4.1.2Peak signal to noise ratio

It measures the quality of a watermarked signal. This performance metric uses to determine perceptual transparency of the watermarked signal with respect to original signal. Firstly, the mean square error is computed which is used as an input to calculate psnr value. The smaller the value of PSNR implies the quality of image is poor.

The PSNR can be calculated as:

$$
P S N R=10 \cdot \log _{10}\left(\frac{M A X_{i}}{M S E}\right)^{2}
$$

$=20 . \log _{10}\left(\frac{M A X}{\sqrt{M S E}}\right)$

$=20 \cdot \log _{10}\left(\mathrm{MAX}_{\mathrm{I}}\right)-10 \cdot \log _{10}(\mathrm{MSE})$

Where MAX denotes the maximum image pixel value

4.1.3Maximum difference

It can be expressed as follows:

$$
M D=\operatorname{Max}\left[\left|x(m, n)-x^{\wedge}(m, n)\right|\right]
$$

The smaller value implies that the image quality is good.

4.1.4Normalized absolute error(NAE)

$$
N A E=\sum_{m=1}^{M} \sum_{n=1}^{N}\left|x(m, n)-x^{\wedge}(m, n)\right| / \sum_{m=1}^{M} \sum_{n=1}^{N}|x(m, n)|
$$

The larger the value of the NAE implies the quality of the image is poor [12].

The Table 1 below shows the experimental results obtained based on the quality parameters used and the analysis of using various $\mathrm{db}$ wavelets

Table 1 DB wavelet analysis using quality parameters

\begin{tabular}{lllll}
\hline $\begin{array}{l}\text { Wavelet } \\
\text { Name }\end{array}$ & MSE & PSNR & MD & NAE \\
\hline db1 & 16.50 & 21.5067 & 1.915 & 0.999 \\
db5 & 16.5088 & 21.5069 & 1.0241 & 0.999 \\
db9 & 16.5088 & 21.51 & 1.0245 & 0.999 \\
db13 & 16.5088 & 21.512 & 1.025 & 0.999 \\
db17 & 16.5088 & 21.51 & 1.0268 & 0.999 \\
db25 & 16.5088 & 21.51 & 1.0268 & 0.999 \\
db29 & 16.5088 & 21.512 & 1.026 & 0.999 \\
db33 & 16.5088 & 21.512 & 1.0259 & 0.999 \\
db41 & 16.5088 & 21.51 & 1.028 & 0.999 \\
db45 & 16.5088 & 21.512 & 1.0276 & 0.999 \\
\hline
\end{tabular}

\section{Conclusion}

Watermark can be in the form of text, logos, images, signatures or numbers. In this paper, an image watermarking technique is proposed which is based on chaotic map logistics map and a DWT transform. Before embedding the watermark chaotic map is used to select random frames to apply on the watermark and then embedded the watermark on chaotic locations. Watermarking technique is highly robust because unauthorized users cannot find the actual location where watermark is embedded. Experimental results reveal that the proposed watermarking technique has good robustness against the many attacks. In the project work, video watermarking technique has been shown highlighting comparative analysis of $\mathrm{db}$ wavelets based on different quality parameters. Each db wavelets is applied on the randomly selected frames by using 
chaotic logistic map from the input coloured video using random number that works as a key for the proposed extraction algorithm. Output shows that not all wavelets are suitable only 45 wavelets $12 \mathrm{db}$ wavelets were applicable for watermarking. The original watermark image and the extracted watermark image are then used as the against various quality parameters to check if the imperceptibility of the watermark is retained after watermark extraction. The proposed watermarking scheme is imperceptible against various quality parameters such as Peaksignal-to-noise ratio, Mean-square error, maximum difference, and normalized absolute error.

\section{Acknowledgment}

None.

\section{Conflicts of interest}

The authors have no conflicts of interest to declare.

\section{References}

[1] Ahuja R, Bedi SS. Video watermarking scheme based on candidates I-frames for copyright protection. Indonesian Journal of Electrical Engineering and Computer Science. 2017; 5(2):391-400.

[2] Wang W, Men A, Chen X. Robust image watermarking scheme based on phase features in DFT domain and generalized radon transformations. In image and signal processing. CISP'09. 2nd international congress on 2009 (pp. 1-5). IEEE.

[3] Dixit A, Dixit R. A review on digital image watermarking techniques. International Journal of Image, Graphics and Signal Processing. 2017; 9(4):5666.

[4] Dixit A, Sharma P, Kulshreshtha V. Blind video watermarking based on DWT-SHUR and optimized firefly algorithm. International Journal of Computer Applications. 2016;147(1):30-6.

[5] Bajracharya S, Koju R. An improved DWT-SVD based robust digital image watermarking for color image. IJEM-International Journal of Engineering and Manufacturing (IJEM). 2017; 7(1):49-59.

[6] Xia XG, Boncelet CG, Arce GR. Wavelet transform based watermark for digital images. Optics Express. 1998; 3(12):497-511.

[7] Dey N, Roy AB, Dey S. A novel approach of color image hiding using RGB color planes and DWT. arXiv preprint arXiv:1208.0803, International Journal of Computer Applications 2012; 36(5):19-24.

[8] Yantao Z, Yunfei M, Zhiquan L. A robust chaos-based DCT-domain watermarking algorithm. In computer science and software engineering, international conference on 2008 (pp. 935-8). IEEE.

[9] Voyatzis G, Pitas I. Chaotic mixing of digital images and applications to watermarking. In proceedings of european conference on multimedia applications, services and techniques (ECMAST'96) 1996 (pp. 68795).
[10] Lu CS, Liao HY. Structural digital signature for image authentication: an incidental distortion resistant scheme. IEEE Transactions on Multimedia. 2003; 5(2):161-73.

[11] Munir R. A chaos-based fragile watermarking method in spatial domain for image authentication. In intelligent technology and its applications (ISITIA), international seminar on 2015 (pp. 227-32). IEEE.

[12] Vora VS, Suthar AC, Makwana YN, Davda SJ. Analysis of compressed image quality assessments, International Journal of Advanced Engineering \& Application, 2010:225-9.

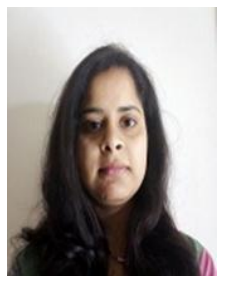

Bhavna Sharma was born in Uttar Pradesh India in 1990. She received her B.Tech in CSE from R.T.U Kota, Rajasthan, India in 2012.Currently, she is doing M.Tech in CSE from S.I.T (Affiliated to A.K.T.U, Uttar Pradesh), India. She is working on the project Digital Image Watermarking Using Logistic Map. Her interest areas are Digital Image Processing, Operating Systems, and DBMS.

Email: sbhavna2812@gmail.com

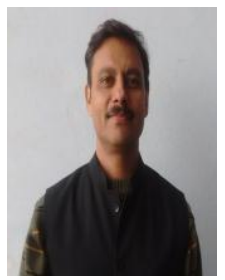

Dr. Pankaj Sharma has a wide experience of twenty years in industry, academics and research. He has done his BE degree in 1997, M.Tech in 2008 and Ph.D in year 2015. He has published 11 research papers in International Journals, 2 research papers in International conferences, and 6 research papers in National Journal and one Book. Presently he is working as Professor \& Head, Department of Computer Science, Sachdeva Institute of Technology, Mathura. His areas of interest are Network Security, Neural Networks, Computer Networks and Advanced Computer Architecture.

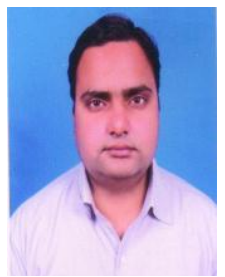

Vyom Kulshreshtha born in year 1985. His native place is Agra, Uttar Pradesh. He has done his B.Tech in Information Technology in 2007 from U.P. Technical University, Lucknow. He completed his M.Tech from Rajiv Gandhi Technical University in year 2013. He is having ten years of experience in Industry and academics. Presently he is working as Assistant Professor in Department of Computer Science at Sachdeva Institute of Technology, Mathura. He has published 6 research papers in International Journals, and 5 research papers in National Journal. His key research fields are Digital Image Processing, Soft computing Techniques and Artificial Intelligence. 